ORIGINAL ARTICLE

\title{
Therapeutic impact of percutaneous spinal biopsy in spinal infection
}

\author{
J J Rankine, D A Barron, P Robinson, P A Millner, R A Dickson
}

Postgrad Med J 2004;80:607-609. doi: 10.1136/pgmj.2003.017863

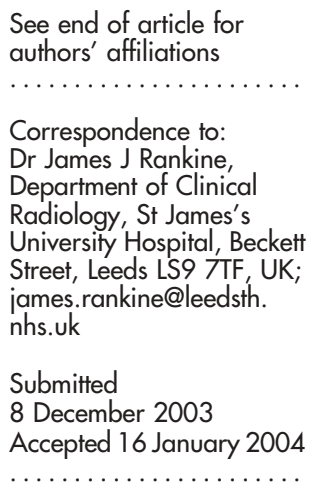

Objective: To investigate the therapeutic impact of percutaneous spinal biopsy in patients with suspected spinal infection.

Design and patients: A review of the case notes and imaging features of 36 patients who underwent percutaneous spinal biopsy was performed. From this group 20 patients with a prebiopsy diagnosis of spinal osteomyelitis were identified. Management before biopsy was noted including the use of antimicrobial therapy. The results of the histology and microbiology were noted along with the subsequent diagnosis and management.

Results: Eight of the 20 patients (40\%) had received antibiotics before the biopsy. An organism was isolated in $8 / 20$ cases (40\%). Of the eight patients on antibiotics, two grew an organism (25\%), including one case of candida in a patient receiving flucloxacillin. Out of 12 patients not on antibiotics there were six cases where an organism was isolated (50\%). The result of the biopsy led to a change in management in seven of the 20 patients (35\%).

Conclusions: Many clinicians are treating spinal osteomyelitis empirically with antibiotics before biopsy, but this reduces the chance of isolating an organism and determining antibiotic sensitivity. Despite this biopsy led to a change in management in $35 \%$ of cases.
$\mathrm{P}$ ercutaneous spinal biopsy is a well recognised technique used in the diagnosis of malignant and infective conditions of the spine. The technical and diagnostic accuracy of the procedure has been extensively investigated. ${ }^{1-3}$ The technique is highly accurate in obtaining a histological diagnosis in cases of malignant disease. ${ }^{4}$ In cases of spinal infection the reported accuracy is more variable with an organism isolated in $40 \%-73 \%$ of cases, ${ }^{5}$ with staphylococcus accounting for $76 \%$ of isolates.

The procedure is not without risk and therefore clinical governance issues mandate that it has a significant impact on the management of the patient. The aim of this study is to investigate the therapeutic impact of percutaneous spinal biopsy in patients with spinal infection.

\section{PATIENTS AND METHODS}

A retrospective study was performed in all patients who had percutaneous spinal biopsies performed for suspected spinal infection of the thoracic and lumbar spine over a three year period. All biopsies were undertaken on an inpatient basis and were performed by one of two consultant radiologists, or a specialist registrar under direct supervision of the consultant. Both consultants specialise in musculoskeletal radiology with a particular interest in spinal disease and provide radiological services for a regional spinal unit.

All case notes were obtained and systematically examined using a pro forma. All patients had a full history and physical examination recorded at the time of their admission. All clinic letters and, where relevant, referral letters from other hospitals were scrutinised. Results of the following were recorded: blood cultures, full blood count, urea and electrolytes, and C-reactive protein. The diagnosis and management before biopsy was noted including any antimicrobial therapy at the time of biopsy.

All patients underwent magnetic resonance imaging of the spine before biopsy. The whole of the spine was covered with sagittal Tl and T2-weighted sequences. Axial Tl and T2weighted sequences were used through areas of abnormality and all patients had sagittal and axial Tl-weighted postgadolinium sequences through abnormal areas.

The biopsies were performed under local anaesthetic with intravenous benzodiazepine sedation (titrated dose of 5$10 \mathrm{mg}$ of diazepam). Fluoroscopy or computed tomography was used for guiding the biopsy. Biopsy was performed using an 11 gauge bone biopsy needle of the Jamshidi type, ${ }^{6}$ using a Cook bone biopsy set (Cook Incorporated, Bloominston, USA). The needle was directed to the intervertebral disc, the end plate, and adjacent vertebral body. Four cores of tissue were obtained and samples sent for microbiological and histological analysis.

A note of biopsy complications was made. The results of the histological and microbiological analysis of the specimens were noted along with the subsequent diagnosis and management with clinical follow up over a minimum period of one year.

\section{RESULTS}

Percutaneous spinal biopsy was performed in 36 patients over a three year period. In 16 patients the clinical and radiological diagnosis prior to biopsy was malignant disease and these patients were excluded from subsequent analysis. This left 20 patients with a prebiopsy diagnosis of spinal infection. There were 13 males and seven females, mean age 53, median 57, range 17 months to 79 years.

In 13 patients $(65 \%)$ there were relevant features in the previous medical history, which may have predisposed to infection. Five patients had undergone surgery in the previous six months. In two cases infection complicated surgery, one case of wound sepsis in a patient who had undergone a coronary artery bypass graft, and one case of an infected femoral nail, inserted for a femoral fracture. The other surgical procedures performed in the six months before biopsy were aortofemoral bypass grafting, right hemicolectomy, and L4 lumbar laminectomy with L4/5 fusion and posterior instrumentation. In the latter case the infection involved the $\mathrm{L} 4 / 5$ intervertebral disc, although there had been 
no surgical procedure performed on the disc. The other relevant features in the history were non-insulin dependent diabetes, rheumatoid arthritis, carcinoma of the oesophagus, bladder carcinoma, paraplegia with urinary self catheterisation, pneumonia, intravenous drug abuse, and a history of contact with tuberculosis. A delay in diagnosis from the onset of symptoms was frequently encountered, in one case up to four months.

The white cell count was raised in $6 / 20$ patients $(30 \%)$. Creactive protein was available in $13 / 20$ patients, mean 89 , range 9-252 (normal value less than 10 ). In only $1 / 13$ cases the C-reactive protein was not raised, a 17 month old child where the pathology revealed chronic inflammation in keeping with chronic osteomyelitis but no organism was isolated.

Blood cultures were positive in six patients; two grew Staphylococcus aureus, one Escherichia coli, and one coagulase negative staphylococcus.

Eight patients $(40 \%)$ had antibiotic therapy before biopsy. Six patients were on flucloxacillin, one on clindamycin, and one patient on antituberculous chemotherapy (after a history of contact with tuberculosis and a positive Heaf test).

Spinal infection occurred in the lumbar spine in 15 cases and in the thoracic spine in five cases. Biopsy was performed under fluoroscopy guidance in 19 cases and one case of infection at T8/9 was performed under computed tomography guidance.

Two patients experienced nerve root irritation for three months after the biopsy, requiring the use of oral analgesia. In both cases the symptoms settled. In one of these cases neural tissue was seen in the histology specimen indicating that a nerve root had been biopsied. One patient died 48 hours after the biopsy from a cerebrovascular accident that was unrelated to the biopsy.

Positive microbiology was obtained in $8 / 20$ patients $(40 \%)$. Table 1 lists the organisms that were isolated.

Of the eight patients on antibiotics before the biopsy only two had positive bacteriology. One patient on flucloxacillin grew candida and one patient on flucloxacillin and fusidic acid grew streptococcus. Of the 12 patients not on antibiotics an organism was isolated in six cases.

\section{Therapeutic impact}

The result of the biopsy led to a change in management in $7 / 20(35 \%)$ of patients. In two cases the bacteriology result led to specific antimicrobial therapy. In one case candida was isolated in an intravenous drug abuser and in the other tuberculosis was isolated. In three cases staphylococcus was isolated which led to starting appropriate antibiotic therapy.

In two cases pathology revealed unsuspected malignancy. In one of these cases there was clear evidence of infection with discitis and epidural abscess. $S$ aureus was isolated from a blood culture, the patient was biopsied before antibiotic therapy and an organism was not isolated from the spinal biopsy: the pathology revealed that the patient also had myeloma. In the second case of unsuspected malignancy, pathology revealed a poorly differentiated carcinoma. The bacteriology in this case revealed a coagulase negative

\section{Box 1: Spinal infection}

- Chronic condition with insidious symptoms. There is frequently a delay in diagnosis of many months.

- There is often a predisposing risk factor.

- Blood inflammatory markers are usually raised.

- Staphylococcus aureus is responsible for $80 \%$ of cases.

\section{Box 2: Spinal biopsy}

- An organism is isolated in only $40 \%$ of cases.

- Patients will frequently have been started on antimicrobial therapy before biopsy, but this reduces the chances of isolating an organism.

- Empirical treatment is directed at Staphylococcus aureus, but it is important to exclude tuberculosis.

- Malignancy is a predisposing factor for spinal infection, so malignancy and infection can coexist.

staphylococcus, though it could be argued that this was a commensal contaminate.

\section{DISCUSSION}

Percutaneous spinal biopsy is generally a safe technique but like other interventional procedures it is not risk-free. Pneumothorax, ${ }^{7}$ paravertebral haematoma, ${ }^{8}$ paraplegia, and even death ${ }^{9}$ have all been reported as complications. It is self evident that the procedure should only be performed when it is likely to alter the management of the patient significantly. It is well recognised as a sensitive technique for obtaining histology in malignancy but has variable results in spinal infection. With $76 \%$ of cases of spinal infection caused by $S$ aureus $^{5}$ there could be an argument for empirical treatment without biopsy.

It is not our departmental policy to start antibiotics prior to biopsy but eight of the 20 patients were on antibiotics before biopsy. Our department is a tertiary referral centre for spinal diseases and it is evident from this study that many centres are practising empirical treatment. Not all departments offer a percutaneous spinal biopsy service and a delay of several months from the onset of symptoms to diagnosis is common. Empirical antibiotic therapy before biopsy is not without its problems as the growth rate fell from $40 \%$ overall to $25 \%$ if the patients were already on antibiotics. The general difficulty in isolating organisms from bacteriological cultures in patients on antibiotics is well recognised.

The intervertebral disc is relatively avascular and this is thought to explain the occurrence of chronic low grade infection and stopping the antibiotic before biopsy is unlikely to influence the ability to isolate an organism, at least in the short term. This raises the question of whether a patient already on antibiotics should be biopsied. The isolation of candida in a patient on flucloxacillin emphasises the need to biopsy patients even if they are already taking antibiotics. The biopsy can be viewed as a technique not only to isolate staphylococcus but to identify the $20 \%$ of cases where another organism is involved. In two of the 20 cases $(10 \%)$ we identified an organism requiring specific antimicrobial chemotherapy: one candida and one tuberculosis. The patient with candida was an intravenous drug abuser, which is a good clinical clue that unusual organisms maybe involved. Tuberculosis was isolated in a patient with a history of recent

Table 1 Organisms identified by microscopy and/or culture

\begin{tabular}{ll}
\hline Organism & No of cases \\
\hline Coagulase positive staphylococcus & 3 \\
Coagulase negative staphylococcus & 2 \\
AAFB (tuberculosis) & 1 \\
Candida albicans & 1 \\
Streptococcus & 1 \\
\hline AAFB, alcohol and acid fast bacilli.
\end{tabular}


travel to the Indian subcontinent who had a paraspinal collection. There are a number of imaging features encountered in spinal tuberculosis that include relative preservation of the intervertebral disc with features more of osteomyelitis than discitis, paraspinal abscesses, and involvement of the posterior elements. ${ }^{10}$ Antituberculous chemotherapy carries significant side effects and many months of treatment are required so there is an understandable reluctance to commence treatment without bacteriological confirmation. Tuberculosis is notoriously difficult to isolate and can take many weeks to culture. While not encountered in this series, we have previously found the demonstration of granulomas on the pathology specimen useful indirect evidence of tuberculosis involvement in cases where bacteriology has failed to isolate it. Another potentially useful technique is the polymerase chain reaction, a laboratory process that allows amplification and detection of mycobacterial DNA from clinical samples. It can be helpful in providing a rapid diagnosis of tuberculosis when microscopy and culture are negative. ${ }^{11}$

In 13 patients $(65 \%)$ there were relevant features in the previous medical history, which may have predisposed to infection. The route of infection is usually haematological, occurring during an episode of bacteraemia, but in one case infection followed a spinal surgical procedure. Discitis after an invasive spinal procedure is rare, occurring with an incidence of $0.2 \% .{ }^{12}$ In these cases the route of infection can be by direct contamination of the spine during surgery. In addition there may be the complicating factor of spinal surgical metalwork. In our case the spinal infection was successfully treated without removing the metalwork.

The demonstration of unsuspected malignancy in two cases emphasises the importance of sending samples to both microbiology and histology laboratories. In one case spinal infection occurred in a patient with unsuspected myeloma. Malignant diseases and their treatment lead to immunosuppression and the authors frequently encounter cases where spinal infection was misdiagnosed as malignant disease in patients with a known malignancy.

The second case of unsuspected malignancy, in retrospect, did not show features characteristic of infection, though there was clearly abnormality of the intervertebral disc. Cases do occur where the imaging diagnosis is not clear cut in differentiating malignancy and infection, again emphasising the need to send samples for bacteriology and pathology.
In summary, this series has demonstrated that a high percentage of patients with spinal infection are being treated empirically with antibiotics before biopsy and this is likely to reduce the chances of subsequent isolation of an organism and appropriate treatment according to antibiotic sensitivity. This may encourage chronicity and further complications such as abscess formation, pathological fractures, and progressive deformity. Despite this spinal biopsy led to a direct change in management in 35\% of patients and is still worthwhile even if the patient has been started on antibiotics. Spinal biopsy should be performed before starting antibiotics with samples sent to both pathology and bacteriology.

\section{Authors' affiliations}

J J Rankine, D A Barron, P Robinson, Department of Clinical Radiology, St James's University Hospital, Leeds, UK

P A Millner, R A Dickson, Department of Orthopaedic Surgery, St James's University Hospital, Leeds, UK

\section{REFERENCES}

1 Stoker DJ, Kissin CM. Percutaneous vertebral biopsy: a review of 135 cases. Clin Radiol 1985;36:569-77.

2 Fouquet B, Goupille P, Gobert F, et al. Infectious discitis diagnostic contribution of laboratory tests and percutaneous discovertebral biopsy. Rev Rhum Engl Ed 1996;63:24-9.

3 Stringham DR, Hadjipavlou A, Dzioba RB, et al. Percutaneous transpedicular biopsy of the spine. Spine 1994;19:1985-91.

4 Shaltot A, Michell PA, Betts JA, et al. Jamshidi needle biopsy of bone lesions. Clin Radiol 1982;33:193-6.

5 Borowski AM, Crow WN, Hadjipavlou AG, et al. Interventional radiology case conference: the University of Texas Medical Branch. Percutaneous management of pyogenic spondylodiskitis. AJR Am J Roentgenol 1998; 170:1587-92.

6 Jamshidi K, Swaim W. Bone marrow biopsy with unaltered architecture: a new biopsy device. J Lab Clin Med 1971;77:335-42.

7 Murphy WA, Destouet JM, Gilula LA. Percutaneous skeletal biopsy in 1981: a procedure for radiologists. Results, review and recommendations. Radiology 1981;139:545-9.

8 Ottolenghi CE. Diagnosis of orthopaedic lesions by aspiration biopsy: results of 1061 punctures. J Bone Joint Surg Am 1955;58:1167-8.

9 Nagel DA, Albright JA, Keggi KJ, et al. Closer look at spinal lesions: open biopsy of vertebral lesions. JAMA 1965;191:975-8.

10 Narlawar RS, Shah JR, Pimple MK, et al. Isolated tuberculosis of posterior elements of spine: magnetic resonance imaging findings in 33 patients. Spine 2002;27:275-81

11 Sritharan V, Barker RHJ. A simple method for diagnosing $M$ tuberculosis infection in clinical samples using PCR. Mol Cell Probes 1991;5:385-95.

12 Silber JS, Anderson DG, Vaccaro AR, et al. Management of postprocedural discitis. Spine J 2002;2:279-87. 